\title{
General Dermatology
}

\section{An explanation for the mysterious distribution of melanin in human skin}

- a rare example of asymmetric (melanin) organelle distribution during mitosis of basal layer progenitor keratinocytes

N. Joly-Tonetti ${ }^{1}$, J.I.D. Wibawa ${ }^{2}$, M. Bell ${ }^{2}$, D.J. Tobin ${ }^{1 * \$}$

${ }^{1}$ Centre for Skin Sciences, Faculty of Life Sciences, University of Bradford, Bradford, West

Yorkshire, UK. ${ }^{2}$ Walgreens Boots Alliance, Nottingham, UK.

*Correspondence: Professor Desmond J Tobin, Centre for Skin Sciences, University of Bradford, Bradford BD7 1DP, West Yorkshire, Britain. d.tobin@bradford.ac.uk Tel : 01274233585

\$ New address : Charles Institute of Dermatology, School of Medicine, University College Dublin

Belfield, Dublin 4, Ireland E: director.charles@ucd.ie T: + 35317166261

Presented as an oral presentation at the 47th Annual Meeting of the European Society for Dermatological Research, September 2017 Salzburg, Austria.

Running Head: Basis of melanin distribution in the human skin epidermis

Manuscript Word count :

Tables :

Figures :

The work described in this study was funded by a research grant to DJT from Walgreens Boots Alliance. M. Bell and JID Wibawa were involved in the study design, data analysis and manuscript preparation. 
The authors have no conflicts of interest to disclose.

\section{What's already known about this topic?}

- Synthesized by the melanocyte, melanin pigment is transferred to surrounding keratinocytes, where it commonly assembles as UVR-protective supra-nuclear caps in the basal layer.

- Melanin presence appears markedly decreased in the epidermis layers above the proliferative basal layer.

- A clear explanation of this classical histologic appearance has been elusive, except via the unproven dogma of 'melanin-degradation' in the supra-basal epidermis.

\section{What does this study add?}

- We propose that the restriction of the vast majority of melanin to the stratum basale results from an asymmetric distribution of melanin granules to the non-differentiating daughter progeny cell during keratinocyte mitosis.

- If required (e.g. during skin regeneration) this shifts to a symmetric mode of melanin inheritance into both daughter cells for more general melanin distribution throughout the epidermis.

- This study reports a rare example of asymmetric organelle distribution during mitosis in human cells. 


\section{What is the translational message?}

- A greater understanding of how melanin is processed within proliferating versus

differentiating keratinocytes in the human epidermis could lead to improved treatment of pigmentary disorders. 


\section{ABSTRACT}

Background: Melanin is synthesized by melanocytes in the basal layer of the epidermis. When transferred to surrounding keratinocytes it is the key UVR-protective biopolymer responsible for skin pigmentation. Most melanin is observable in the proliferative basal layer of the epidermis, and only sparsely distributed in the stratifying/differentiating epidermis. The latter has been explained, despite formal evidence, to 'melanin degradation' in supra-basal layers. Objectives: Our aim was to re-evaluate this currently-accepted basis for melanin distribution in the human skin epidermis, and whether this pattern is altered after a regenerative stimulus. Methods: Normal epidermis of adult human skin, at rest and after tape-stripping, was analysed by a range of (immuno)histochemical and high-resolution microscopy techniques. In vitro models of melanin granule uptake by human keratinocytes were attempted.

Results: We propose a wholly different fate for melanin in the human epidermis. Our evidence indicates that the bulk of melanin is inherited only by the non-differentiating daughter cell post mitosis in progenitor keratinocytes, via asymmetric organelle inheritance. Moreover, this preferred pattern of melanin distribution can switch to a symmetric or equal daughter cell inheritance mode under conditions of stress including regeneration. Conclusions: We provide in this preliminary report a plausible and histologically-supportable explanation for how human skin pigmentation is efficiently organized in the epidermis. Steady state epidermis pigmentation may involve much less redox-sensitive melanogenesis than previously thought, and at least some pre-made melanin may be available for re-use. The epidermal-melanin unit may be an excellent example to study organelle distribution via asymmetric or symmetric inheritance in response to micro-environment and tissue demands. 
One Sentence Summary: Melanin fate follows an unexpected asymmetric organelle distribution during the mitosis of progenitor keratinocytes in human skin epidermis. 


\section{INTRODUCTION}

The turnover, differentiation, stratification and desquamation of the epidermis contributes to formation of an indispensable skin barrier that protects us from dehydration and external insults among other key functions. This structure is maintained by cell dynamics that involve high levels of proliferation from progenitor keratinocytes $(\mathrm{KC})$ located in the stratum basale. In order to maintain and support this essential barrier, humans have further evolved strategies to protect the proliferative cells of the epidermis against damaging effects of ultraviolet radiation (UVR). A key developmental strategy was the development of a UVR-screening pigmentation system that is both continuatively-active and facultatively-adaptive. ${ }^{1}$

In this way, skin melanization represents a compromise solution to the conflicting physiological needs of (folate) photoprotection and vitamin- $\mathrm{D}_{3}$ synthesis ${ }^{2}$ Like many aspects of skin phenotype, pigmentation quality influences social behaviour, and human dyspigmentation conditions (e.g. pigment loss in vitiligo, or pigment gain in melasma or with ageing) can be psychologically debilitating. Thus, improved models of human skin pigmentation disorders are urgently needed, given the rather limited utility of murine models where hair follicle-restricted melanocytes are used as a proxy for the human epidermal-melanin unit. ${ }^{1}$

\section{MATERIALS AND METHODS}

Donor skin samples: Formalin-fixed, paraffin-embedded (FFPE) and fresh frozen skin of healthy adult donors (phototype III-VI; $\underline{\mathrm{n}=10}$ ) were obtained with donor consent and ethics committee approval. Plaque psoriasis skin samples (phototype II-IV; $\underline{\mathrm{n}=4}$ ) were kindly provided by Prof. Rachel Watson from University of Manchester (UK). 
Volunteer study: The study was submitted and approved by the Ethics Committee of the University of Bradford (UK) and included SPT VI African geographic ancestry volunteers $(n=4)$. Skin from sun-protected upper inner arm was tape-stripped 15-40 times using D-Squame Stripping Discs (D-101, CuDerm). This procedure did not induce extensive redness or pruritus in these donors. After 48 to 72 hours the skin was anesthetized with lidocaine 2\% (Mercury Pharma Intl Ltd) and a $0.3 \mathrm{~mm}$ punch biopsy was taken by a registered surgeon. Skin samples were immediately fixed in $10 \%$ formalin for 1 day prior to paraffin embedding or were frozen immediately in OCT-sucrose ${ }^{3}$

Histochemistry: Routine H\&E staining was performed on 7-8 $\mu \mathrm{m}$ FFPE sections. Modified Warthin-Starry staining was performed on $5 \mu \mathrm{m}$ FFPE sections and $3 \mu \mathrm{m}$ frozen sections, as described. ${ }^{4}$

Immunohistochemistry of FFPE Sections: Paraffin-embedded section were cut at $4 \mu \mathrm{m}$ and stained for mitosis-related marker expression, including phospho-H3 Ser10 (\#9701, Cell Signalling; ab47297, abcam), Insc (ab102953, abcam), Par-3 (ab64840, abcam), and KIF-16B (sc390309, Santa Cruz). Secondary antibodies used were donkey anti-rabbit (A-21206; A-21207, Life Technologies), donkey anti-mouse (A-21202, Life Technologies) and donkey anti-goat (A11058, Life Technologies) IgG.

Immunohistochemistry of Frozen Sections (IHC-F): Frozen sections were cut on a cryostat at 7 $\mu \mathrm{m}$ and air-dried for 20 minutes. Sections were fixed with acetone or $4 \%$ paraformaldehyde in PBS pH 7.4 for 10 min, rinsed with PBS and blocked in 10\% normal donkey serum (D9663, Sigma-Aldrich) for $1 \mathrm{~h}$. Primary antibodies were diluted in block solution and incubated for $1-2 \mathrm{~h}$ at room temperature or overnight at $4^{\circ} \mathrm{C}$. After rinsing with PBS-tween20 0.1\% (PBS-T), 
secondary antibodies were added for 1-2h at room temperature. After rinsing with PBS-T, sections were incubated for $1 \mathrm{~min}$ in $300 \mathrm{nM}$ DAPI solution (62248, ThermoFisher), rinsed with PBS before mounting in Vectashield medium ( $\mathrm{H}-1400$, Vector Laboratories).

Co-localisation IHC-F: Tissues were double-probed with anti-LAMP2 and anti-LC3 antibodies. Five images per donor were marked into five supra-nuclear areas of interests. Pearson's correlation coefficients and Spearman's rank correlation were calculated using either Coloc2 tool in Fiji/ImageJ (v1.49) or JACoP plugin on ImageJ (v1.48).

High-resolution light microscopy and transmission electron microscopy: Normal skin samples from three SPT III donors were fixed in half-strength Karnovsky's fixative as 3-mm ${ }^{3}$ tissue cubes. The tissues were post-fixed in $2 \%$ osmium tetroxide and embedded in araldite resin, as described previously. ${ }^{5}$ Semithin and ultrathin sections were cut with a Reichart-Jung microtome (Leica $\mathrm{GmbH}$, Wetzlar, Germany); the former were stained with the metachromatic stain, toluidine blue/borax, examined by light microscopy and photographed (Leitz, Germany). Ultrathin sections were stained with uranyl acetate and lead citrate and were examined and photographed using a JEM-1200EX (Jeol, Tokyo, Japan) electron microscope.

Cell culture. Human primary KC from juvenile foreskin were purchased (C-12005, PromoCell, UK) and cultivated in KGM2 (C-20111, PromoCell, UK). For bead uptake study, $0.5 \mu \mathrm{m}$ fluorescent beads (Fluoresbrite ${ }^{\circledR}$ BB, YG or YO Carboxylate Microspheres, Polysciences) were added to cell culture medium at a final concentration of $1.456 \times 10^{8}$ particles. $\mathrm{ml}^{-1}$. After 2 days incubation all keratinocytes incorporated beads, as evidenced by cytometric analysis. To avoid misinterpreting recently-phagocytosed elements, keratinocytes were transferred to bead-free medium 2 days before analysis i.e. observing bead distribution pattern during mitosis. 
Preparation of supramolecular crowded (SMC) matrix: To stimulate polarity in cultured human $\mathrm{KC}$ to attempt recapitulation of asymmetric melanin distribution during mitosis, supramolecular crowded (SMC) matrix was made. ${ }^{6,7}$ Briefly, normal human dermal fibroblasts were cultivated in DMEM FBS $10 \%$ supplemented with $200 \mu \mathrm{g} \cdot \mathrm{ml}^{-1}$ of ascorbic acid-2 phosphate, $37.5 \mathrm{mg} \cdot \mathrm{ml}^{-1}$ of

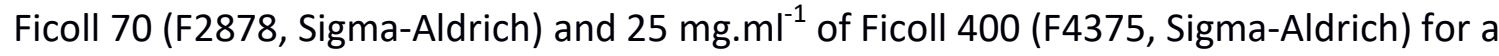
minimum of 2 weeks allowing matrix proteins deposition and maturation. Fibroblasts were removed with $0.5 \%$ sodium deoxycholate for $30 \mathrm{~min}$ on ice. The matrix was rinsed with PBS before seeding the $\mathrm{KC}$.

Immunocytochemsitry-Frozen (ICC-F). Bead-laden primary KC were seeded at a density of 5,000 to 40,000 cells $/ \mathrm{cm}^{-2}$ in Nunc ${ }^{\circledR}$ Lab-Tek $^{\circledR}$ Chamber Slide $^{\mathrm{TM}}$ (C7182 Sigma-Aldrich) either directly on plastic, collagen IV (C-7521, Sigma-Aldrich) or SMC matrix. Cells were fixed in 4\% paraformaldehyde in PBS pH 7.4 for 10 min at room temperature, permeabilized in PBS $0.1 \%$ Triton X-100, rinsed several times with PBS and blocked in 10\% normal donkey serum (D9663, Sigma-Aldrich) for 30 min. Primary antibodies were diluted in block solution and incubated for $1 \mathrm{~h}$ at room temperature or overnight at $4^{\circ} \mathrm{C}$. After rinsing with PBS, secondary antibodies were added for $1 \mathrm{~h}$ at room temperature. After rinsing with PBS, sections were incubated for 1 minute in 300 nM DAPI solution (62248, ThermoFisher), rinsed briefly with PBS before mounting (H-1400, Vector Laboratories). For analysis of the distribution pattern of the beads, cells were fixed just before DAPI staining and mounting.

Cytometry. The percentage of KC incorporating fluorescent beads was analyzed by flow cytometry with a Beckman Coulter - CyAn ${ }^{\mathrm{TM}}$ ADP analyser (Beckman Coulter, UK) equipped with Summit ${ }^{\mathrm{TM}}$ Software, version 4.2. For each sample, 10,000 events were collected. Briefly, bead- 
laden $\mathrm{KC}$ were seeded in several wells of a 6-well plate in KGM2 without beads. One fraction of the seeded cells was analyzed directly and served as a control. Two and five days later, cells were trypsinized and the beads content of the cell population was analyzed. Experiments were performed using plastic or collagen-IV treated plates (C-6745, Sigma-Aldrich).

Epidermis equivalent reconstruction. 3D skin equivalent models were made using Poumay's

method. ${ }^{8,9}$ Bead-laden KC were seeded on a polycarbonate insert (PIHP01250, Merck Millipore) at a density of 200,000 cells $/ \mathrm{cm}^{-2}$ in a differentiation medium made with $K G M 2$ supplemented with $\mathrm{CaCl}_{2}$ to reach a calcium concentration of $1.5 \mathrm{mM}$. The medium also contained $50 \mu \mathrm{g} \cdot \mathrm{ml}^{-1}$ of ascorbic acid-2 phosphate (A8960, Sigma-Aldrich) and 1\% of lipid mixture 1 (L0288, SigmaAldrich). One day later, the inserts were lifted at the air-liquid interphase on top of a steel mesh in a Petri dish filled with $100 \mathrm{ml}$ of the differentiation medium, and cultivated for 14-17 days without medium change. For one experiment, a SMC matrix made with fibroblasts was used. Microscopy. Bright field and epifluorescence images were taken with a Nikon eclipse 80i with a Nikon DS-5Mc. Confocal images were taken with a LSM510 Zeiss confocal microscope. Image analysis and treatment was done with ImageJ (1.47v).

\section{RESULTS}

The French dermatologist Farabeuf first reported that the vast preponderance of epidermal melanin appears to be concentrated in the stratum basale of the human epidermis, with only a sparse distribution of melanin in the stratum spinosum to stratum corneum ${ }^{10}$ (Figure. 1a, unstained). Similar melanin distribution occurs in the epidermis of other mammals. ${ }^{11}$ The full 
distribution and incidence of melanin granules in the human steady-state epidermis can be confirmed using the much-neglected Warthin-Starry staining of melanin in the epidermis (Figure 1b), as this histochemical stain is more specific for melanin than Fontana-Masson stain. ${ }^{4}$ On higher magnification view, using high resolution light microscopy, we show that much of this melanin is organised within melanin-containing phagolysosomes (melano-phagolysosomes) that congregate to the apical pole of basal keratinocytes as so-called melanin micro-parasols (Figure 1b\&c). We found no evidence to suggest that this marked difference in melanophagolysosome density is related to significant differences in cell density between the basal and supra-basal layer (see Figure 1c) where broadly similar numbers of KC nuclei appear for any given length of epidermis in these two layers. The paucity/near absence of melanin granules in the stratum granulosum and stratum corneum of Caucasian skin was demonstrated by transmission electron microscopy (Figure 1d). This remarkable partitioning of melanin distribution is even more striking in the hair follicle infundibulum (Figure. 1e), where suprabasal KC layers were seen to be essentially melanin-free. This pattern of melanin segregation is also maintained even in hyper-proliferative skin diseases like psoriasis (data not shown).

The incidence and distribution of melanin granules in the healthy epidermis of 11 Caucasian females (SPT II-III) was assessed by scanning 2D vertical sections post Warthin-Starry melanin staining (Figure 1b). When the percentage of skin section area with melanin was set at an arbitrary $100 \%$, over $70 \%$ of total melanin was found to be restricted to the stratum basale (Figure 1f \& g). Furthermore, when considered as fraction of melanin in the stratum basale, only $18 \%$ and $10 \%$ were detected in stratum spinosum/granulosum and stratum corneum 
respectively. Thus, $\mathrm{KC}$ in the stratum basale contain up to 10 -fold more melano-phagolysomes than KC in the immediate adjacent first layer of the stratum spinosum.

In an effort to explore a basis for this so-called melanin 'disappearance' already by the first supra-basal layer of the epidermis, we revaluated the long-held dogma that this disappearance can be attributed to melanin 'degradation'.

As a report suggests that Caucasian KC 'degrade' melanin more efficiently than do African KC, ${ }^{12}$ based on an autophagy-based pathway. ${ }^{13}$

We first examined to what extent this autophagic pathway for melanin degradation exists in normal human epidermis, by assessing the expression of autophagolysosomes in the epidermis. Specifically, we evaluated the distribution of the lysosome marker LAMP2, especially when this was co-localised with the key autophagy marker LC3. While LAMP2 was readily detected (Figure 2a), and was correlated with the degree of pigmentation of the tissue (due to the lysosomal nature of the membrane encompassing the melanin granules when in keratinocyte), the autophagy LC3 expression was only weakly detected. Moreover, when we attempted to colocalise LAMP2 and LC3 in areas where melanin was identifiable, we observed no significant colocalisation between the two proteins (i.e. Pearson's correlation coefficient and Spearman's rank correlation not significantly different from zero) (Figure $\mathbf{2 b}$ ) in this melanogenic compartment.

These results therefore encouraged us to conduct a deeper assessment of how the wellappreciated histological distribution patterns of melanin could be explained. 
Thus, we were interested to determine the basis for an apparent paradox that must balance this striking retention of melanin to within the stratum basale, while at the same time acknowledge the obvious processes of proliferation/turnover of KC progeny in the stratum basale, and their subsequent differentiation to drive upward stratification for barrier formation. ${ }^{14}$ Using skin from adult African geographic-ancestry donors we investigated the fate of melanin during $\mathrm{KC}$ mitosis in the stratum basale. It was exceptionally difficult to characterize, morphologically, sufficient numbers of mitotic events/figures, due to their intrinsic rarity at any one moment in time. ${ }^{15}$ Of 62 mitotic events observed many were not interpretable, due to being either suprabasal or in cells with little melanin. Of 14 unambiguous mitotic events, 13 were vertical asymmetric and 1 bipolar (horizontal division). Thus, we probed the epidermis for the expression of the histone histone-H3 (3a.c), which is specifically phosphorylated throughout all mitosis stages. ${ }^{16}$ We observed KC progenitors undergoing vertical mitosis, where melanin granules redistributed to the basal pole of dividing cells. As mitosis progressed, melanin granules segregated asymmetrically and was observed in samples taken from donors included in this study. Thus, melanin was distributed en masse to the daughter $\mathrm{KC}$ retained within the stratum basale (Figure 3a-d). By contrast, the second daughter cell destined to leave the stratum basale (for differentiation through upward stratification) lacked significant amounts of melanin.

To further increase the frequency of witnessing melanin-segregating mitotic occurrences, we tape-stripped the skin of healthy African-ancestry volunteers to stimulate elevated KC mitotic 
activity in the stratum basale. ${ }^{17}$ Mildly-stripped skin samples (15-20 tapes) yielded more frequent mitosis events compared with unstripped skin, while not inducing cell/tissue damage (Figure 4a-c). Melanin distribution was concentrated to the basal layer, similar to un-tape stripped skin. Again we mostly observed an asymmetric distribution of melanin during mitosis of basal KC progenitors, with the confirmatory re-positioning of melanin to the basal pole of the dividing cell (Figure 4c). However, increasing tape-stripping strength further ( 40 tapes) resulted not only in significant loss of stratum corneum, parakeratosis, and some pycnosis (Figure 4d) there was also a marked redistribution/dilution of melanin across the three lower layers of the epidermis (Figure 4e). Melanocytes retained their expected distribution (Figure 4f), which concurs with previous students on the impact of tape stripping on melanocytes. ${ }^{18}$ This tissue regeneration stimulus was associated with very many $\mathrm{KC}$ mitotic events (e.g., up to $20 \mathrm{KC}$ divisions across a $0.3 \mathrm{~mm}$ length of epidermis) throughout the lower three $\mathrm{KC}$ layers, where mitoses in supra-basal (non-basement membrane bound) exhibited a melanin distribution that switched to a symmetric mode. Both daughter cells inherited a similar melanin granule loading (Figure 4g,h). Of 150 observed events, 50 events were interpretable, and of these $70 \%$ exhibited symmetric melanin distribution to both daughter cells. Specifically, we observed prophase and metaphase mitotic figures in which melanin granules were scattered evenly throughout the KC cytoplasm. At anaphase most melanin distributed to the cell equator causing melanin granules under these conditions to partition symmetrically between both daughter cells (Figure $\mathbf{4 g}$ ). The phenotypic consequence of this switch to a symmetric melanophagolysosome distribution was a striking dilution of melanin intensity to the adjacent 2-3 supra-basal KC in addition to the more proximal KC layers (Figure 4e). 
Next, we attempted to explore the potential mechanism for asymmetric organelle/melanin granule distribution during $\mathrm{KC}$ in mitosis in the human epidermis, by examining whether this could be associated with the phenomenon of divergent centrosome filiation, i.e. whether or not the melanin-laden daughter cell inherits the old 'mother' centrosome during mitosis.

Asymmetric cell division in mouse neuroblasts is associated with same-generation centrosomes positioning to the same daughter cell. ${ }^{19}$ Importantly, there is increasing evidence that elements of the mitotic machinery, including the centrioles, participate in regulating the fate decision of which daughter cell inherit different age organelles. ${ }^{20,21}$ Moreover, it was recently shown that 'inappropriate peroxisome segregation' during KC mitosis can influence cell division, skew daughter fate and disturb epidermal balance. ${ }^{22}$ Thus, we aimed to see if there was any correlation between the filiation of the centrosome and the amount of melanin inherited by a daughter KC. In other words, whether mother and daughter centrosomes were always associated with one type of daughter KC. We attempted to characterize the expression of the mother centriole appendage protein, cenexin (ODF2, HGNC:8114) in KC 2D monolayers in vitro. We found that cenexin expression level discriminated maternal from daughter centriolederived centrosomes (Figure 5a). We also observed multiple positive cenexin immuno-reactive spots in the probed KC, but felt on balance that these were not always consistent with centrosome location during mitosis (Figure $\mathbf{5 b}$ ), and concluded that loss of KC polarity here may be responsible for these equivocal findings.

We next assessed different 2D and 3D skin models to attempt a recapitulation of asymmetric melanin distribution during $\mathrm{KC}$ mitosis in vitro. To stimulate polarity in cultured human $\mathrm{KC}$, 
these cells were seeded onto so-called supramolecular crowded (SMC) matrix derived from normal dermal fibroblasts. ${ }^{6,7}$ Fluorescent beads can be taken up by $\mathrm{KC}^{23}$ and in our study these were used as a melanin granule proxy. These were found to be rapidly phagocytosed and packed within KC phagolysosome (Figure 5c), and distributed eccentrically in a pattern resembling KC melanin supra-nuclear caps in vivo. From prophase onward however, the beads were found scattered throughout the KC cytoplasm (Figure 5d), thereafter concentrating at the equator of the cell during anaphase, before finally distributing symmetrically between both daughter cells during telophase (Figure 5e). Only symmetric bead distribution was ever detected using this model, as was confirmed by a single unique peak shift in cytometric analysis of bead content in KC (Figure 5f).

Next we interrogated a 3D epidermis reconstruct that was established with fluorescent beadladen KC. Basal KC were found to be polarised in the early 3D model, with beads concentrated above the cell nucleus (as in vivo). However, with progressive stratification the bead-associated fluorescence was not retained within the basal layer but instead re-distributed to supra-basal layers (Figure $\mathbf{5 g}$ ) before ultimately delaminating.

\section{DISCUSSION}

In this research, which is at its early stages, we help to resolve a fundamental enigma underpinning our successful adaptation to terrestrial life, i.e. fate of melanin in human skin. In our effort to explain the striking difference in melanin occurance between the stratum basale and superbasal epidermis in human skin we interrogated our proposal/hypotensis by assessing the value of some alternative potential explanations for these anatomic feature. Regarding a 
putative difference in term of cell density in the different strata of the epidermis, we conclude that unlikely contributes significantly to the observed melanin gradient in the epidermis. This analysis concurs with the work of Droste ${ }^{24}$ where total numbers of melanin granules per cell were tracked throughout the epidermis using the gold standard of transmission electron microscopy and across different ethnicities. Furthermore, the currently popular consideration of autophagy-mediated degradation of melanin during the epidermal differentiation is also most unlikely to explain this distribution of melanin. First, no formal evidence of melanin degradation in any living system has ever been reported. This long-held teleological dogma has gained some (albeit weak) circumstantial support, based on the recognised lysosomal nature of melanosomes, and their derivative the melano-phagolysosome, within the melanin-accepting KC. ${ }^{25,26}$ Thus, as others have contended before ${ }^{23,27}$ and most recently ${ }^{26}$ we conclude that only very low expression of autophagy-associated machinery exist in normal healthy human steadystate epidermis. Where present, this is not likely to be involved in regulating the epidermalmelanin unit in the basal and immediate supra-basal layer. Moreover, we previously demonstrated that melanin content is largely unaltered in autophagy-deficient melanocytes. ${ }^{28}$ Significantly, the literature reports that only melanosome lipid and protein fractions degrade, and then using in vitro approaches. ${ }^{29}$ Crucially the melanin indole biopolymer itself does not degrade. In fact, melanin biopolymer degradation is possible only with harsh laboratory oxidative and acidic conditions $\mathrm{s}^{30}$ and attempts to degrade melanin in vitro with lysosomal extracts have strikingly failed. ${ }^{31,32}$ Some ligninolytic fungi may degrade melanin, but only under conditions inconsistent with epidermis physiology. ${ }^{33}$ 
We found that most melanin is preferentially retained in the stratum basale. Our results raises a number of very interesting and important questions. What is the melanocyte's role in melanin-handling dynamics by proliferating KC, given the melanocyte's post-mitotic status in the healthy steady-state epidermis? Can melanin in the basal layer be reused/recycled, and if so how long can it persist? Could reuse of melanin act like a brake on excessive melanogenesis in neighbouring melanocytes? There is no histological evidence that the observed melanin gradient in human epidermis is related to changes in $\mathrm{KC}$ (nor phagosome) densities in the basal and supra-basal layer, and so concurs with the extraordinary and remarkable stability of the

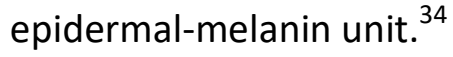

We tried to reproduce this asymmetric distribution of melanin in vitro in 2D and 3D. Our culture model failed to sufficiently approximate KCs in in vivo human epidermis. This drift toward a symmetric distribution of melanin-proxy (beads) with culture age highlights limitations of our current models for accurate assessment of melanin distribution dynamics. Future work could also address if blocking of mitosis in vitro to check subsequent melanin/bead inheritance and to further address more fully the relationship between mitosis and melanin segregation.

It could be envisaged that melanocytes themselves influence this differential melanin gradient distribution by somehow selectively transferring higher rates of melanin to adjacent basal cells rather than suprabasal KC. However, such a view is not consistent with our understanding of the epidermal-melanin unit, ${ }^{34}$ the fate decision of basal KC progenitors, ${ }^{35}$ and that the KC drives many aspects of melanocyte function, including melanogenesis. Still it will be important to investigate more fully the melanocyte contribution to this process using for example full- 
thickness pigmented skin equivalents. We speculate that melanin distribution between KC daughter cells is a key element in maintaining a stable epidermal-melanin unit, where unfettered levels of redox-labile melanogenesis may be undesirable. We would also like to confirm whether centrosome filiation drives asymmetric distribution of melanin granules, by favouring segregation of melanin to the daughter $\mathrm{KC}$ with the older/mother centrosome. For this study we are developing a full-thickness pigmented human skin equivalent, which retains full polarity of the epidermal melanin unit. The second main finding of this study suggests that during times of tissue stress (tissue regeneration or wound-healing) a switch from steady-state asymmetric to symmetric melanin distribution may be advantageous to drive melanin into a hyper-activated Malphigian layer (Figure 6a). More widely distributed melanin may confer greater protection (e.g., anti-microbial) during times of stress. In summary, our findings suggest an alternative, refined model to the melanin degradation dogma that significantly improves our understanding of the processes involved in human pigmentation (Figure $6 \mathbf{b}$ ). It should be noted that melanin distribution in the epidermal-melanin unit differs markedly from melanin fate in the skin's follicular melanin unit. ${ }^{36,37}$ In the latter case melanin is transferred to immature precortical keratinocytes, and these cells retain the melanin granules intact as they differentiate into fully mature hair shaft cortex keratinocytes. In this way melanin granules move continually during anagen to fully pigment the elongating hair shaft. ${ }^{36,37}$ Still, an in-depth comparison of the epidermal-melanin unit and the follicular melanin unit in the same tissue, could provide a most accessible way to compare how cells of the same histological type (i.e. melanocytes and keratinocytes) process melanin in quite distinct ways during cell proliferation and differentiation. It is quite likely the at least part of this difference relates to the lack of direct 
contact between UVR and the follicular melanin unit in the anagen bulb (which can be as much as $6 \mathrm{mms}$ deep in human scalp, by comparison with the superficial distribution of the epidermal $\underline{\text { melanin unit. It will also be important to investigate whether UVR stress results in a similar }}$ switch from asymmetric to symmetric melanin distribution that we observed here with tape stripping stress, and similarly drives melanin granules into the upper layers of the epidermis, where they can provide more optimal protection to viable keratinocytes. This would explain the reported change in the distribution of melanin from the lower to mid epidermis post UVR, which was seen to be more dramatic in those with darker skin. ${ }^{38}$

Our lab is currently extending investigations of how melanin distribution may be modulated between asymmetric and symmetric modes within the human epidermis. We feel this is an important pursuit, not least given our recent findings that melanin distribution in the epidermis may have implications for protection against both skin carcinogenesis and actinic damage associated with photo-aging. ${ }^{39}$ 


\section{CONFLICTS OF INTEREST}

The authors state no conflict of interest.

ACKNOWLEDGMENT:

This study was funded by a research grant to DJT from Boots UK. 


\section{REFERENCES}

1 Slominski A, Tobin DJ, Shibahara S, Wortsman J. Melanin pigmentation in mammalian skin and its hormonal regulation. Physiol Rev 2004; 84(4):1155-228

2 Slominski A, Postlethwaite AE. Skin Under the Sun: When Melanin Pigment Meets Vitamin D. Endocrinology 2015; 156(1):1-4

3 Tobin DJ. The cell biology of human hair follicle pigmentation. Pigment Cell Melanoma Res 2011: 24(1):75-88

4 Joly-Tonetti N, Wibawa JID, Bell M, Tobin DJ. Melanin fate in the human epidermis: a reassessment of how best to detect and analyse histologically. Exp Dermatol 2016;25(7):501-4

5 Tadokoro T, Yamaguchi Y, Batzer J et al. Mechanisms of skin tanning in different racial/ethnic groups in response to ultraviolet radiation. J Invest Dermatol 2005; 124(6):1326-32

6 Ang XM, Lee MHC, Blocki A et al. Macromolecular crowding amplifies adipogenesis of human bone marrow-derived mesenchymal stem cells by enhancing the pro-adipogenic microenvironment. Tissue Eng Part A 2014; 20(5-6):966-981

7 Chen C, Loe F, Blocki A et al. Applying macromolecular crowding to enhance extracellular matrix deposition and its remodeling in vitro for tissue engineering and cell-based therapies. Adv Drug Deliv Rev 2011; 63(4-5):277-90

8 Frankart A, Malaisse J, De Vuyst E et al. Epidermal morphogenesis during progressive in vitro 3D reconstruction at the air-liquid interface. Exp Dermatol 2012; 21(11):871-5

9 Poumay Y, Dupont F, Marcoux S et al. A simple reconstructed human epidermis: preparation of the culture model and utilization in in vitro studies. Arch Dermatol Res 2004; 296(5):203-11

10 Farabeuf LH. De l'épiderme et des épithéliums [Internet]. Paris: Georges Masson; 1872 [cited 2014 Dec 30]. Available from: http://gallica.bnf.fr/ark:/12148/bpt6k6207521r

11 Morales-Guerrero B, Barragán-Vargas C, Silva-Rosales GR et al. Melanin granules melanophages and a fully-melanized epidermis are common traits of odontocete and mysticete cetaceans. Vet Dermatol 2017; 28(2):213-e50

12 Ebanks JP, Koshoffer A, Wickett RR et al. Epidermal keratinocytes from light vs. dark skin exhibit differential degradation of melanosomes. J Invest Dermatol 2011; 131(6):1226-33

13 Murase D, Hachiya A, Fullenkamp R et al. Variation in Hsp70-1A Expression Contributes to Skin Color Diversity. J Invest Dermatol 2016; 136(8):1681-91 
14 Zanet J, Freije A, Ruiz M et al. A Mitosis Block Links Active Cell Cycle with Human Epidermal Differentiation and Results in Endoreplication. PLOS ONE. 2010; 5(12):e15701

15 Thuringer JM. Studies on cell division in the human epidermis. II. A. Rate of cell division in the prepuce. B. Influence of various factors on cell division. Anat Rec 1928; 40(1):1-13

16 Hendzel MJ, Wei Y, Mancini MA, Van Hooser A, Ranalli T, Brinkley BR, et al. Mitosis-specific phosphorylation of histone $\mathrm{H} 3$ initiates primarily within pericentromeric heterochromatin during $\mathrm{G} 2$ and spreads in an ordered fashion coincident with mitotic chromosome condensation. Chromosoma 1997; 106(6): 348-360

17 Pinkus H. Examination of the Epidermis by the Strip Method. J Invest Dermatol 1952;

19(6):431-47

18 Bartosik J, Stender IM, Kobayasi T, Agren MS. Ultrastructural alteration of tape-stripped normal human skin after photodynamic therapy. Eur J Dermatol. 2004; 14(2):91-5.

19 Ouellet J, Barral Y. Organelle segregation during mitosis: Lessons from asymmetrically dividing cells. J Cell Biol 2012; 196(3):305-13

20 Moore DL, Jessberger S. Creating age asymmetry: consequences of inheriting damaged goods in mammalian cells. Trends Cell Biol 2017; 27(1):82-92

21 Coelho M1, Tolić IM. Asymmetric damage segregation at cell division via protein aggregate fusion and attachment to organelles. Bioessays. 2015;37(7):740-747.

22 Asare A, Levorse J, Fuchs E. Coupling organelle inheritance with mitosis to balance growth and differentiation. Science 2017; 355(6324)

23 Wolff K, Konrad K. Melanin pigmentation: an in vivo model for studies of melanosome kinetics within keratinocytes. Science 1971; 174(4013):1034-5 
24 Droste MS. Determining Factors of Skin Colouration: A Light and Electron Microscopic Study of the Distribution of Melanin and Its Degradation in the Human Epidermis. Universität Hamburg; 2006.

25 Toda K, Pathak MA, Parrish JA et al. Alteration of Racial Differences in Melanosome Distribution in Human Epidermis after Exposure to Ultraviolet Light. Nature 1972; 236(66):1435

26 Hurbain I, Romao M, Sextius P et al. Melanosome distribution in keratinocytes in different skin types: melanosome clusters are not degradative organelles. J Invest Dermatol. 2018;

138(3):647-656

27 Lee HM, Shin DM, Yuk JM et al. Autophagy negatively regulates keratinocyte inflammatory responses via scaffolding protein p62/SQSTM1. J Immunol 2011; 186(2):1248-58

28 Zhang CF, Gruber F, Ni C, Mildner M et al. Suppression of autophagy dysregulates the antioxidant response and causes premature senescence of melanocytes. J Invest Dermatol 2015; 135(5):1348-1357.

29 Otaki N, Seiji M. Degradation of melanomes by lysosomes. J Invest Dermatol 1971; 57(1):1-5

30 Del Bino S, Ito S, Sok J et al. Chemical analysis of constitutive pigmentation of human epidermis reveals constant eumelanin to pheomelanin ratio. Pigment Cell Melanoma Res 2015; 28(6):707-717

31 Borovanský J, Hach P, Smetana K, Elleder M, Matous-Malbohan I. Attempts to induce melanosome degradation in vivo. Folia Biol (Praha) 1999; 45(2):47-52

32 Saito N, Seiji M. Degradation of melanosomes in vitro and in vivo. Pigment Cell 1976. (3):384-392

33 Luther JP, Lipke H. Degradation of melanin by Aspergillus fumigatus. Appl Environ Microbiol 1980; 40(1):145-55

34 Fitzpatrick TB, Breathnach AS. The Epidermal melanin unit system. Dermatol Wochenschr $1963 ; 147: 481-489$

35 Nöske K, Stark HJ, Nevaril L et al. Mitotic Diversity in Homeostatic Human Interfollicular Epidermis. Int J Mol Sci 2016 17(2): pii: E167.

36 Slominski A, Wortsman J, Plonka PM, Schallreuter KU, Paus R, Tobin DJ. Hair follicle pigmentation. J Invest Dermatol. 2005; 124(1):13-21. 
37 Barthel LK, Raymond PA. Improved method for obtaining 3-microns cryosections for immunocytochemistry. J Histochem Cytochem 1990; 38(9):1383-1388

38 Tobin DJ, Fenton DA, Kendall MD. Cell degeneration in alopecia areata. An ultrastructural study. Am J Dermatopathol 1991; 13(3):248-56

39 Fajuyigbe D, Lwin SM, Diffey BL et al. Melanin distribution in human epidermis affords localized protection against DNA photodamage and concurs with skin cancer incidence difference in extreme phototypes. FASEB J 2018 Feb 2:fj201701472R. doi:

10.1096/fj.201701472R. [Epub ahead of print]

\section{FIGURE LEGENDS}

Fig. 1. The preponderance of total epidermis melanin is observable in the stratum basale. Normal SPT VI epidermis, unstained (a) or stained with WS stain (b) displays melanin mainly in the stratum basale. Melanin is typically concentrated as supranuclear caps $(\mathbf{b}, \mathbf{c})$. Supra-basal layers contain much less observable pigment, but some melanin can be additionally detectable with WS stain (c). Note the broadly similar cell densities in these two layers (i.e. 5 nuclei distances). Intact melanin granules are not readily detectable in the upper strata of Caucasian epidermis (d). This paucity of melanin is particularly striking in the lower hair follicle infundibulum (e, arrow, WS stained), where supra-basal layers are essentially melanin-free in this compartment despite stratification and terminal differentiation. Analysis of tissue sectional area consisting of melanin in 11 donors showed some inter-individual variability (f), but on average reveals $>70 \%$ of total detectable melanin of the epidermis in the stratum basale $(\mathbf{g})$. Scale bars a-d,e $=25 \mu \mathrm{M}, \mathrm{f}=2 \mu \mathrm{M}$

Fig. 2. Little evidence that autophagy is prominent in healthy human skin, including melanogenic compartment. Normal skin from upper inner arm (UIA; sun-protected) and dorsal 
forearm (DFA: sun-exposed) were assessed for expression of the lysosome marker LAMP2 and the autophagy marker LC3A/B. LC3 (green) is most widely used maker of auto-phagosomes; central to autophagy, while LAMP2 (red) is major lysosomal protein for lysosome integrity, $\mathrm{pH}$ and catabolism. Co-localization of both markers in mature autophago-lysosomes was rare in our samples. Any co-localised pixels were random localisation as judged by i.e., little evidence of autophagosomes. Pearson's correlation coefficient and Spearman's rank correlation not significantly different from zero. Scale bars $a=25 \mu \mathrm{M}$

Fig. 3. Evidence of asymmetric melanin granule distribution in proliferating basal KC in normal human epidermis. Using the mitosis marker phospho-H3 (green) and nuclear stain DAPI (blue), with artificially-colored melanin (red), is was possible to detect melanin distribution within progenitor $\mathrm{KC}$ in the epidermis $(\mathbf{a}, \mathbf{c})$ and to compare this pattern in parallel with associated bright-field view of the same section area $(\mathbf{b}, \mathbf{d})$. It was also possible to assess the concentration of melanin within defined compartments of the cell (bi,ii). Scale bars a-d $=10 \mu \mathrm{M}$

Fig. 4. Impact of tape-stripping of the epidermis on melanin distribution in human epidermis. Mild stripping preserves the epidermis morphology (a), whereas aggressive tape stripping induces cell edema, pyknosis and parakeratosis (b). Warthin-Starry (WS) stain reveals that mildly-stripped epidermis conserves the intense pigmentation segregation to the stratum basale $(\mathbf{c})$, whereas in more traumatically tape-stripped epidermis melanin is redistributed and diluted to at least 3 innermost layers of the epidermis $(\mathbf{e}, \mathbf{f})$. Note increased parakeratosis (d, white arrows). Asymmetric melanin distribution broadly retained in $\mathrm{KC}$ proliferation after mild 
tape-stripping (c), while aggressive tape stripping was more often associated with symmetric melanin distributions (g,h). Scale bars: $\mathrm{a}-\mathrm{e}=25 \mu \mathrm{m}, \mathrm{f}=15 \mu \mathrm{m}, \mathrm{g}=10 \mu \mathrm{m}, \mathrm{h}=15 \mu \mathrm{m}$.

\section{Fig. 5. In vitro 2D and 3D culture of keratinocytes does not fully recapitulate in vivo} asymmetric distribution of melanin during mitosis.

$(\mathbf{a}, \mathbf{b})$ In vitro and in situ discrimination of mother/daughter centrioles in KC. ODF2/cenexin (marker of 'old mother' centriole at solid arrow-head) during KC mitosis in vitro. Open arrowhead demarcates the 'new mother' centriole (a). By contrast, centriole lineage detection was equivocal in situ with multiple immuno-reactive dots detected (b). Scale bars: $10 \mu \mathrm{m}$. Fluorescent beads (red) used as melanin granule proxy in 2D (c-e) and 3D cultures of KC (f). Beads were engulfed by keratinocytes (blue nuclei) and distribute around cell centrosome (c). During keratinocyte mitosis, beads distribute (apparently randomly) throughout the cell cytoplasm at prophase (d), and gathered in the mid-zone of dividing cells where they appear partitioned in two halves (e). During 3D skin equivalent reconstruction, bead-laden KC divide symmetrically and delaminate to form a stratified epidermis-like structure. (f) Dilution of the melanin-like beads in proliferating keratinocytes in a 2D culture. At day 0 of the analysis, all keratinocytes have incorporated beads (yellow peak) compared to the bead-free control keratinocyte population (red peak). After 2 days (blue peak) and 4 days (green peak) all beadpositive keratinocytes are represented in a single peak, i.e., the shift reflects the beads diluting among the proliferating population of cells. (g) After 14 days, fluorescence is lost from the stratum basale and is mainly present in the upper epidermis near the stratum corneum (f). Scale bars: $5 \mu \mathrm{m}$. 
Fig. 6. Schematic representations of asymmetric and symmetric distribution of melanin in

human epidermis. (a) Cartoon showing the different arrangements of melanin distribution within KC of normal steady-state human epidermis. Melanosomes are produced in the melanocytes (1) and then transferred to surrounding KC, where supra-nuclear melanin caps are seen (2). Progenitor KC division re-distributes this melanin asymmetrically so that most melanin is inherited by the daughter $\mathrm{KC}$ that will replace the progenitor $\mathrm{KC}$ and stay in the basal layer (3). By contrast, a small amount of melanin enters the daughter KC that differentiated and stratifies (4). (b) Cartoon proposing the potential for switching of melanin distribution between daughter cells after progenitor KC proliferation. The steady state epidermis is associated with asymmetric melanin distribution (left), while stress stimuli that induce transient hyperproliferation/ regeneration may switch melanin distribution to symmetric mode where melanin distribution is broadly between both daughter progeny of the proliferating progenitor KC. 

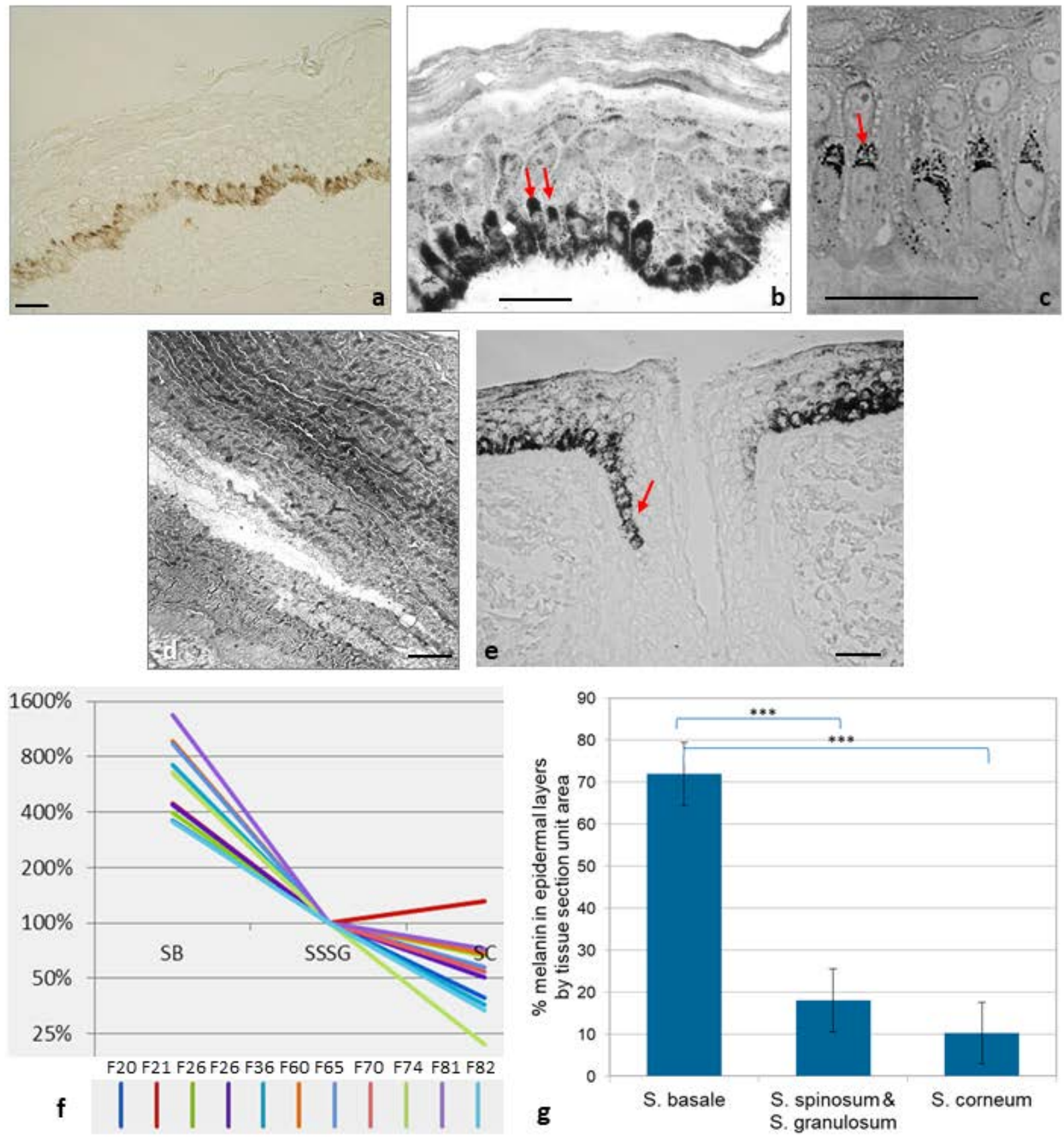


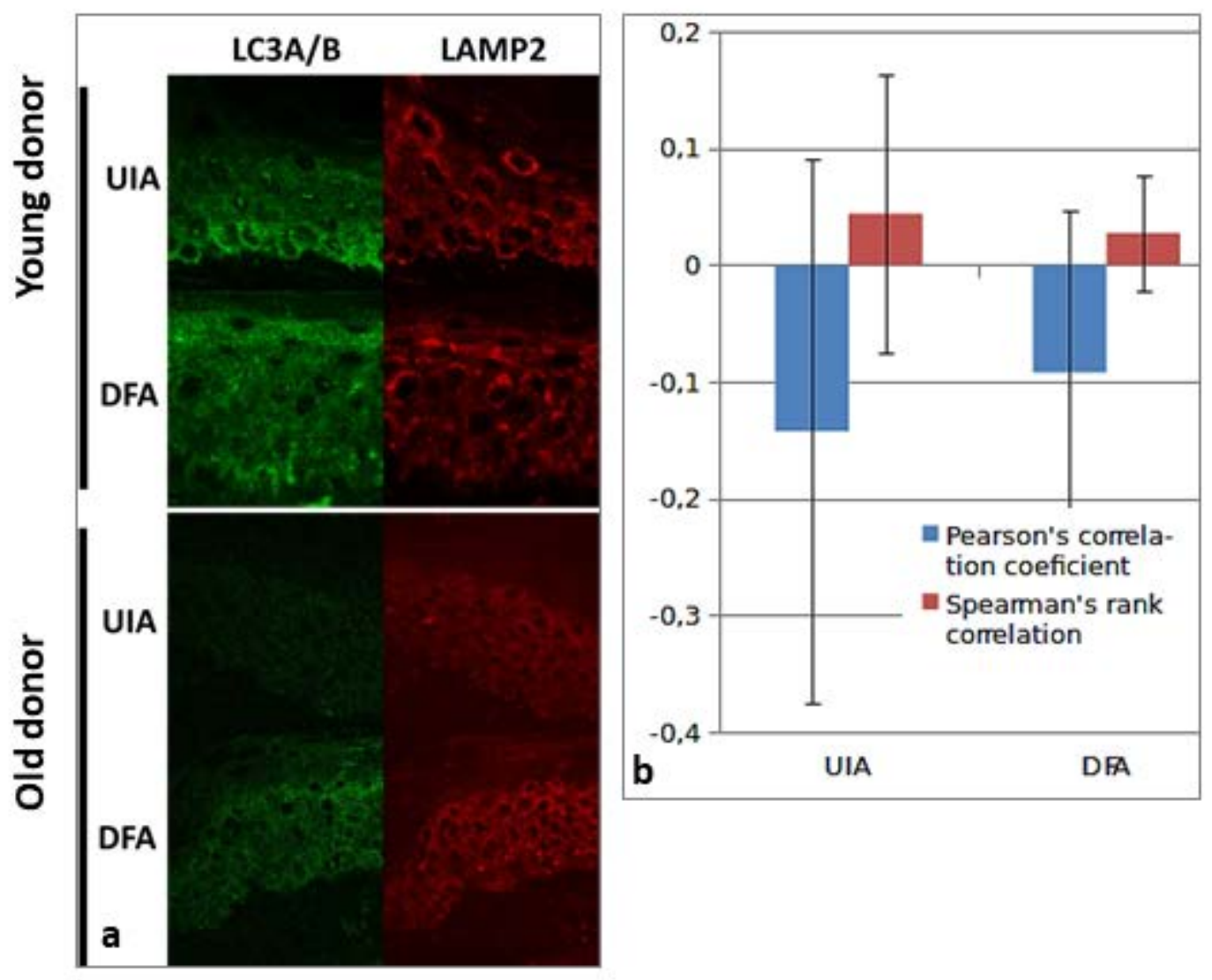



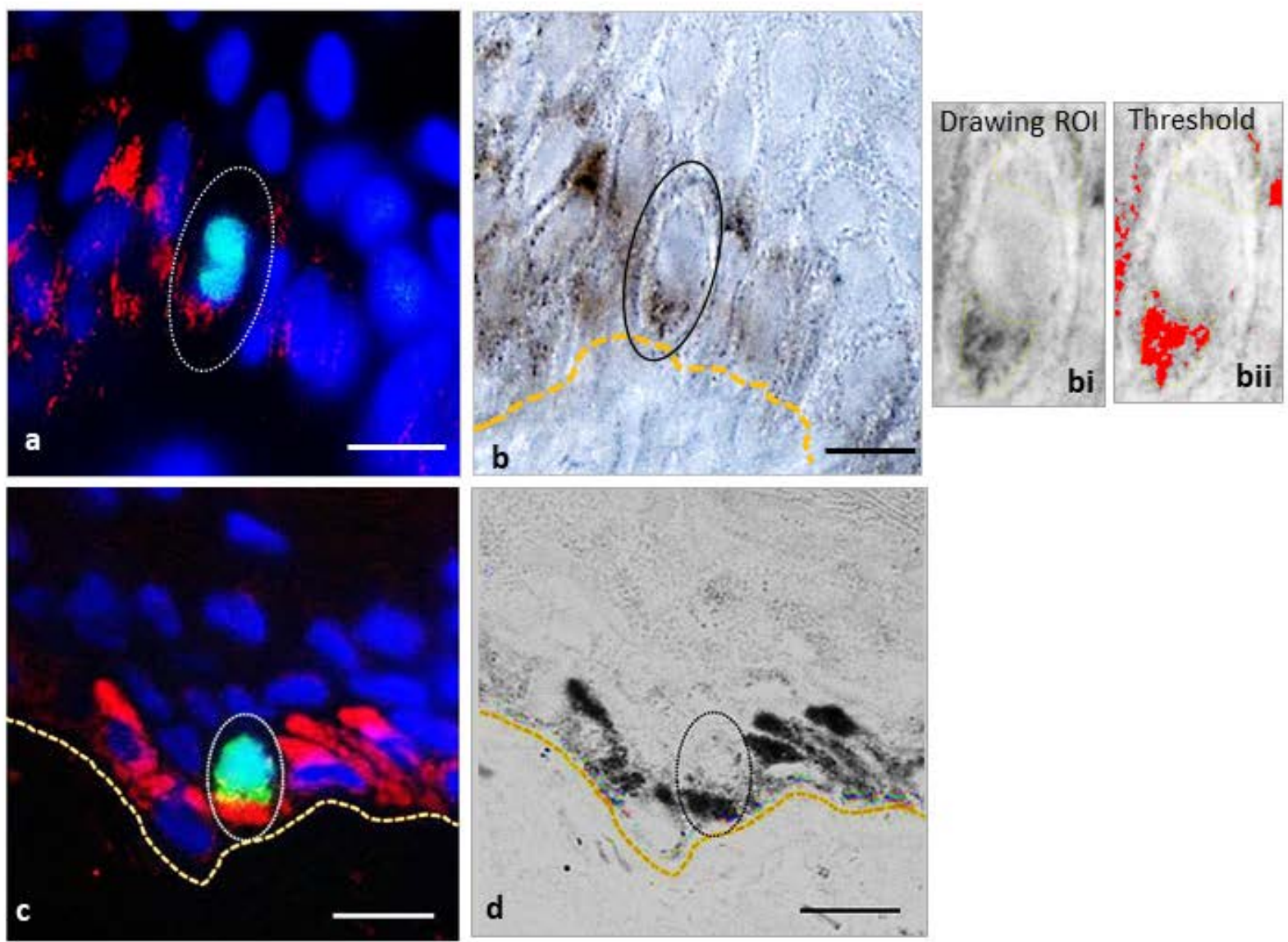

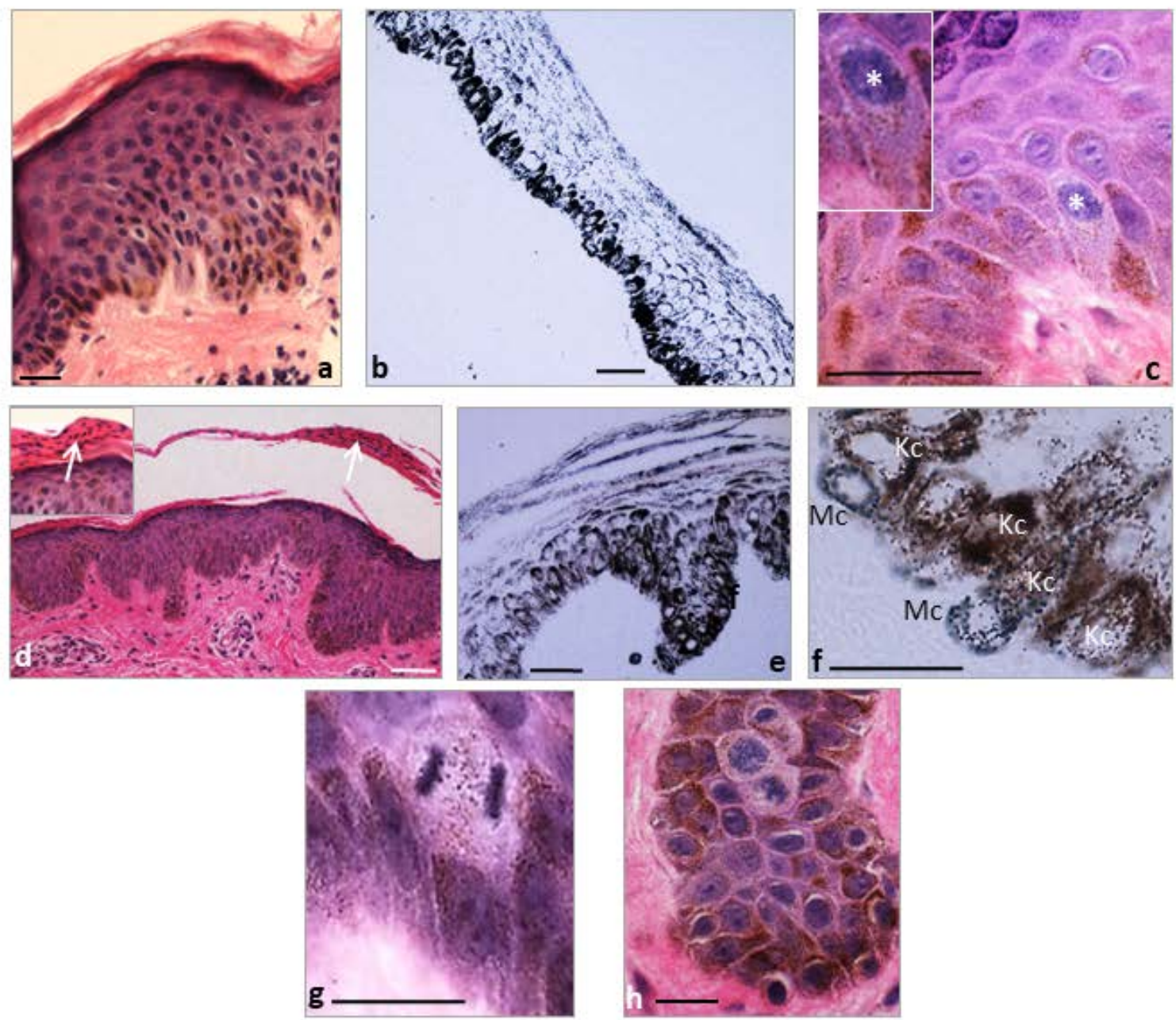


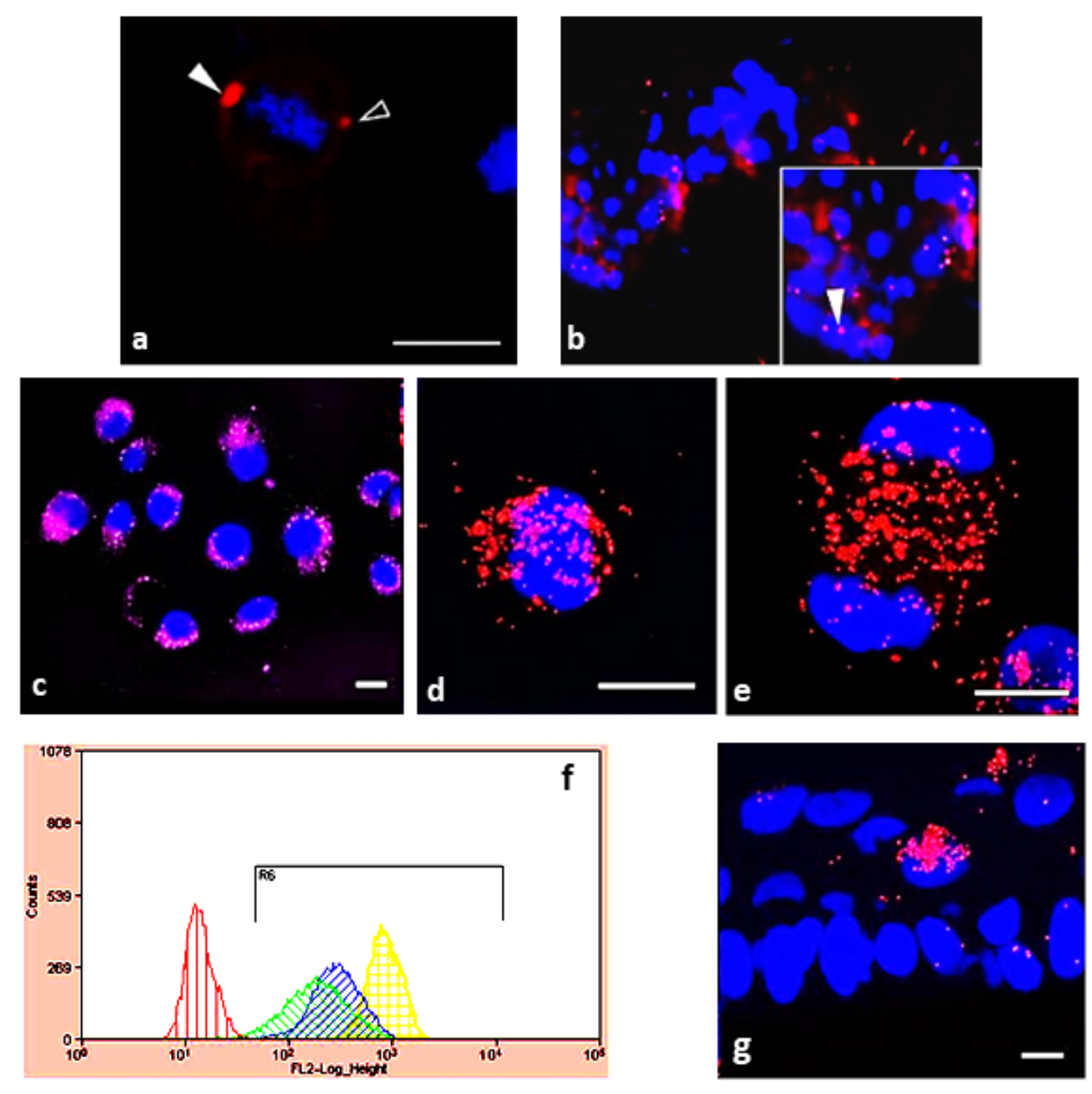




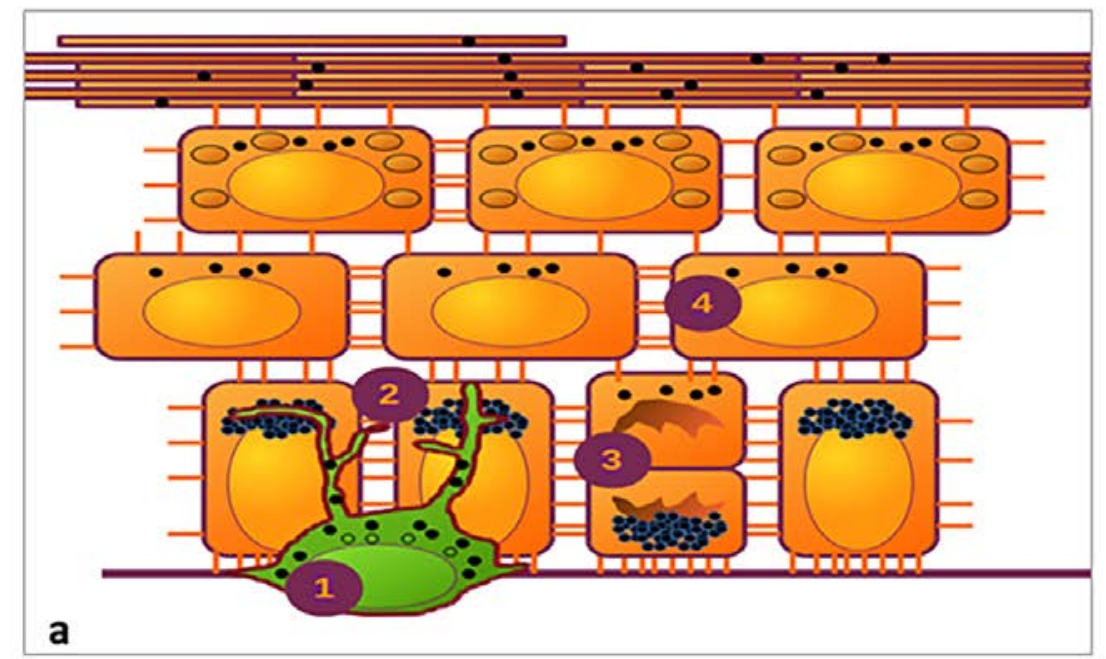

asymmetric

